\title{
La creación frustrada de cátedras de lenguas orientales (egipcio antiguo, asirio y chino) en la Universidad Central
}

\author{
Miguel Á. Molinero Polo \\ Universidad de La Laguna \\ mmolipol@ull.es
}

\begin{abstract}
RESUMEN
En 1899, M. Morayta y Sagrario presentaba una proposición de ley en el Congreso de los Diputados para la creación de tres cátedras de lenguas orientales, en la Universidad Central de Madrid. Éste fue el intento más significativo para establecer una Orientalística académica española en el s. XIX. En el artículo se analizarán sus precedentes en la sociedad española y las diversas causas para el escaso arraigo universitario de la especialidad. El autor considera que éste ha de ligarse más a la insuficiente presencia de historiadores profesionales en España y a la dirección magrebí que tomaba el colonialismo español que a un desinterés o una incapacidad por apreciar la importancia de esas civilizaciones. Se examinan las intenciones del propio texto de la proposición, en especial su pretensión de formar jóvenes en centros académicos europeos para que crearan escuela al regresar a su cargo, así como sus derivaciones académicas y legislativas inmediatas. Se consideran también las implicaciones frente a la actividad de algunos intelectuales del momento. Por último, se argumenta que, medio siglo después de la fracasada reforma legislativa, la Egiptología española ha iniciado su despegue desde instituciones académicas oficiales, en la senda de los objetivos de M. Morayta.
\end{abstract}

Palabras clave: Orientalismo, Egiptología española, Miguel Morayta y Sagrario, José Ramón Mélida Alinari.

\section{The Failed Creation of Oriental Languages' Chairs (Ancient Egyptian, Assyrian and Chinese) in the Universidad Central, Madrid}

\begin{abstract}
In 1899, the member of the parliament and professor of History M. Morayta y Sagrario set a bill before the Spanish Congreso de los Diputados (House of Commons) to create three chairs of oriental languages in the Universidad Central of Madrid. This was the most serious attempt to create a Spanish academic field of studies on Orientalism in the $19^{\text {th }}$ century. In the article, precedents for the creation of a scientific Oriental linguistic discipline in Spain and the causes of its limited presence in Universities are analysed. The author considers that it should be attributed rather to the scarce number of professional historians in the country and to the orientation towards the Magreb of the Spanish colonialism than to the lack of interest on these civilizations. The detail of the bill's text are examined, specially its pretension to instruct young persons in European Faculties to prepare them for organizing a school of Oriental languages when they came back to their posts, and its immediate academic and legislative consequences. As a conclusion it is stated that it is through official academic institutions -and not through private ones- that Spanish Orientalism is sprouting in the last decades in the path pretended by M. Morayta's objectives.
\end{abstract}

Key words: Orientalism, Spanish Egyptology, Miguel Morayta y Sagrario, José Ramón Mélida Alinari.

Gerión

2011, 29, núm 2, 15-33 
En varios estudios sobre la Universidad española se alude, sin especificar detalles, a una ley de cátedras de lenguas orientales en el tránsito entre los siglos XIX y XX1. Paralelamente, recientes trabajos biográficos sobre José Ramón Mélida Alinari afirman que el rechazo de esta disposición legislativa había significado el abandono de su pretensión de ser el primer catedrático español de Egiptología y creador en nuestro país de esta disciplina².

Uno de los aspectos interesantes de estas alusiones es que a partir de ellas podríamos suponer la implicación de instancias oficiales en la creación de una Orientalística académica que, según la segunda referencia, concerniría sobre todo al Egipto antiguo. Más sorprendente aún es que se habría intentado en un momento en que sólo un número muy limitado de naciones europeas había reglamentado el estudio de las lenguas y las civilizaciones de Oriente. Ambas circunstancias son suficientemente significativas para motivar un análisis más profundo de ese proyecto de ley. Estos son los términos con los que en realidad debemos mencionarlo, pues nunca pasó de ese estadio.

\section{Precedentes}

La ley, de haber sido aceptada, no habría sido el primer ensayo del Estado español para activar algún tipo de actuación académica relacionada con la Orientalística antigua. Ninguno fue de gran intensidad, pero estuvieron concentrados, sobre todo, en el Sexenio Revolucionario y en el año de transición hacia la Restauración, por lo que no podemos dejar de reconocer una intencionalidad de presencia internacional en la que el Mediterráneo Oriental estaba presente.

En 1869, una delegación oficial española asistió a las celebraciones organizadas por el Gobierno egipcio con motivo de la apertura del Canal de Suez. Antes de su partida, el Director del Museo Arqueológico Nacional, Ventura Ruiz Aguilera, había propuesto al Director General de Instrucción Pública que una comisión de anticuarios viajara acompañando a la misión diplomática para ver y estudiar las antigüedades de Egipto y, con un permiso del Virrey, adquirir objetos que enriqueciesen las colecciones de la institución. Sin embargo, la Comisión no llegó a formarse ${ }^{3}$.

Este intento fallido debió de estar en el origen de la Comisión Arqueológica a Oriente que viajó a bordo de la fragata Arapiles en el verano de $1871^{4}$. Compuesta por tres miembros, Juan de Dios de la Rada y Delgado, facultativo y jefe de la Sección de Prehistoria y Edad Antigua del Museo Arqueológico Nacional, Jorge Zammit y Romero, como especialista en lenguas e inscripciones y Ricardo Velázquez Bosco, joven arquitecto que acababa de terminar sus estudios en la Escuela. En el nacimiento de la idea tuvieron que estar presentes tanto el recuerdo de la reciente expedición

${ }^{1}$ Por ejemplo, Gutiérrez Cuadrado, 1985: 83-84.

2 Almela Boix, 1991: 132; retomado por Casado Rigalt, 2007: 32; con más detalle y matices: Casado Rigalt, 2006: 133-134.

3 Marcos Pous, 1993: 53.

${ }^{4}$ Chinchilla, 1993. 
de naturalistas al Pacífico ${ }^{5}$ como la experiencia que el propio Rada había adquirido como miembro de las Comisiones Científicas a diversas provincias españolas organizadas desde el Museo. La nave recaló en una docena de puertos orientales desde los que se realizaron pequeños desplazamientos hacia el interior. Los miembros de la Comisión, ayudados por la tripulación, se encargaron de medir y trazar plantas y alzados de monumentos, sacar vaciados de esculturas y relieves, reconocer yacimientos, visitar Museos y colecciones y adquirir -y recibir en regalo- una notable cantidad de piezas. Sin embargo, salvo escasas excepciones, sólo permanecían unas horas en cada puerto y una lamentable penuria económica lastró toda su actividad, imposibilitando un programa más amplio y consecuente de estudio de monumentos y adquisición de objetos.

Adolfo Rivadeneyra, que algunos autores califican como el más importante de los orientalistas españoles del s. XIX, fue enviado por el Gobierno español a Persia en 1874. Ya tenía experiencia en la región por un cargo de vicecónsul en Damasco que había desempeñado de 1869 a 1870. En esta segunda ocasión, su misión era analizar las posibilidades industriales y comerciales de Persia, por donde viajó durante un año. En ambas expediciones, el desempeño de sus obligaciones diplomáticas se vio acompañado de la copia de inscripciones, identificación de yacimientos y descripción detallada de ruinas monumentales así como de la adquisición de un pequeño número de piezas que pasó a los fondos del Museo Arqueológico Nacional. Miembro de varias sociedades geográficas españolas, redactó sendas memorias de estas estancias en Oriente con las que comunicó sus descubrimientos ${ }^{6}$.

La reforma en la década de 1870 de los estatutos de la Escuela de Bellas Artes de Roma abrió la posibilidad de que los pensionados de Arquitectura emprendieran un viaje por Oriente durante su segundo año ${ }^{7}$. En el s. XIX era habitual la implicación de estos técnicos en el estudio de las antigüedades e incluso en su excavación. Pocos becarios se desplazaron a este destino y sin regularidad, la mayor parte a Grecia, pero algunos prolongaron el viaje por Egipto. Así, en 1876-1877, Aníbal Álvarez dibujó varias obras en el Museo de Bulaq mientras que Ramiro Amador de los Ríos, más intrépido, visitó los monumentos del Alto Egipto, compartió vivencias con el Marqués de Rochemonteix, el epigrafista francés que había iniciado la monumental copia integral del templo de Edfú ese mismo año ${ }^{8}$ y residió unos meses en la antigua Tebas; resultado de este viaje fue un estudio del templo de Luxor, hoy perdido. En 1891, fue Alberto Albiñana quien regresó a esta localidad, centrando su labor en el templo de Khonsu que se halla en el recinto de Karnak.

Todas estas actuaciones tienen algo en común: la Orientalística antigua ${ }^{9}$ es secundaria en ellas y se halla sólo como un elemento adicional a un objetivo práctico

${ }^{5}$ www.pacifico.csic.es. López-Ocón (2003: 482) ha calificado esta expedición como el principal intento de construir una ciencia imperial o neo-colonial en el reinado de Isabel II.

${ }^{6}$ Escribano Martín, 2005.

7 García Sánchez, 2004.

8 David, 1994: 243.

9 Como se verá más adelante, el proyecto de ley de lenguas orientales implicaba también la creación de una cátedra de chino. Sin embargo, en este artículo no se incluye un análisis de los precedentes relacionados con el conocimiento hispano sobre el Extremo Oriente. No obstante, es interesante mencionar, como testimonio del carácter de su autor, la obra Historia de la China de Eduardo Toda i 
diferente, diplomático, comercial o ambos a la vez. En la fragata Berenguela tenía que viajar una delegación que ratificase unos acuerdos económicos con Japón, por lo que se aprovechó para que los mismos responsables de la firma estuvieran presentes en la primera travesía por el Canal de Suez durante su inauguración y, con ellos, la comisión de arqueólogos si hubiera sido nombrada. Situación semejante es la de la fragata Arapiles, pues se aprovechaba un viaje ya previsto de finalidad política, la de reconocer "la cultura, industria, progreso moral y material" de los países de Oriente Medio y firmar con ellos tratados diplomáticos; los comisionados se incorporaron en Nápoles cuando la expedición, literalmente, estaba ya en marcha. A. Rivadeneyra viajó a Irán porque poco antes el Gobierno español había sancionado el tratado de amistad con este país y convenía analizar las posibilidades de comercio con él. Sin embargo, la conclusión del diplomático en ese ámbito fue menos positiva que sus hallazgos arqueológicos: consideraba que no había razón para que España se interesase por establecer relaciones económicas con Irán. La opinión del capitán de la fragata Arapiles era la contraria con respecto a los países ribereños del Mediterráneo oriental y lamentaba que no se habían cruzado con un solo barco mercante español en sus aguas; sólo conoce una línea regular de vapores que trasladaban algodón turco desde Esmirna para las fábricas de Cataluña ${ }^{10}$. Así, las puertas que abrían estos pioneros al Orientalismo español al protagonizar unos viajes tan singulares, se cerraban involuntariamente con el dictamen de A. Rivadeneyra, pues no hubo financiación europea para excavaciones en el Oriente del s. XIX sin beneficios paralelos, económicos o políticos, de las naciones que enviaban a sus arqueólogos.

Desde el punto de vista universitario, también en las décadas precedentes se había dado un primer paso con la introducción de una lengua oriental antigua en el plan de estudios, antes que ninguna otra, moderna o incluso peninsular diferente al castellano: la creación de una cátedra de sánscrito en la Universidad Central, en 1875. Su titular tenía que impartir una asignatura voluntaria en el ciclo de doctorado. En realidad, su carácter oriental no tuvo ningún peso en su creación; se hizo por sus conexiones con otras lenguas indoeuropeas y por un espíritu de imitación un tanto superficial, pues los autores extranjeros la mencionaban y las universidades de renombre la impartían $^{11}$. El resultado, en las primeras décadas de impartición, fue un fracaso. Primero, en la propia institucionalización de la cátedra, pues el proceso consistió en elegir a un titular al que se encargó que organizara la docencia durante dos años en los que tenía que crear el programa y escribir una gramática, a pesar de que el decreto reconoce "el estado en que se hallan estos estudios entre nosotros". Segundo, en la cooptación del catedrático, pues en la oposición quedó excluido el único filólogo español que había aprendido sánscrito en el extranjero, Francisco García Ayuso; y en una segunda convocatoria, por ausencia del primer catedrático sólo un par de años después, él mismo formó parte del tribunal y se enzarzó en una disputa pública con los candidatos a los que criticaba sus escasos conocimientos e incluso sus ideas políticas. La labor desa-

\footnotetext{
Güell, 1893, escrita durante la estancia del cónsul en este país. Allí fue trasladado desde Egipto, por cuya civilización también se interesó y escribió sobre ella, mientras ejerció el cargo en El Cairo ( $c f$. infra).

${ }^{10}$ García Tudela, 1873: 19-20, 24, 37, 55.

11 Gutiérrez Cuadrado, 1985: 81.
} 
rrollada por este segundo elegido fue, finalmente, de escaso relieve, lo que Gutiérrez Cuadrado atribuye sobre todo a la falta de un armazón institucional ${ }^{12}$.

No sólo desde instancias oficiales se intentaron abrir vías de acercamiento hacia el Orientalismo. Los beneficios económicos que A. Rivadeneyra consideraba que no podrían producirse en las relaciones con Persia están en la base de la creación, durante las décadas de 1870 y 1880 , de varias Sociedades Geográficas, de Historia Natural, de Africanistas y Colonistas, Antropológicas, etc., a imitación de las que ya existían en los "países civilizados (...) que fomentan y difunden los conocimientos geográficos"13. La pretensión, imbuida de nacionalismo decimonónico, era mantener un cierto nivel de prestigio internacional en el mismo campo en que ya lo desarrollaban otras naciones europeas. La labor de estas sociedades no era inocente. Los exploradores permitían el conocimiento de un territorio que era después integrado por medios militares y explotado a continuación en beneficio de las potencias coloniales. Los mismos arqueólogos no estaban ausentes de esta política, pues se implicaban, por la propia necesidad de su disciplina, en el análisis del territorio y en la administración colonial.

En 1881, Juan Víctor Abargues de Sostén organizaba una expedición científica, geográfica y mercantil al mar Rojo por encargo de la Asociación Española para la Exploración del África, que había sido fundada por Alfonso XII como filial de la creada en Bélgica por Leopoldo II; para ella solicitó material al Ministerio de la Guerra. El desenlace parece contradictorio. Por una parte, un despacho de la legación hispana en El Cairo informaba que el cónsul inglés le había denunciado por presentarse en Abisinia atribuyéndose fraudulentamente un cargo consular, el título de comendador de varias órdenes y ser portador de regalos del rey español ${ }^{14}$. Sin embargo, recogió numerosos datos de interés sobre las regiones visitadas, que plasmó en Notas del viaje (...) por Etiopía, Xoa, Zebul, Uolo, Galas, etc. y presentó una ponencia al Congreso Español de Geografía Colonial y Mercantil de noviembre de 1883 en la que expuso un proyecto de expansión comercial mediante la fundación de una agencia a través de la cual se negociaría con los puertos del Mar Rojo y el Oriente Próximo, para que España se proveyera directamente de los productos de esas regiones de África y Asia a las que exportaría también los suyos. Las representaciones locales aprovecharían el paso de los barcos que servían las líneas a Filipinas a través del canal de Suez, para efectuar el intercambio ${ }^{15}$. Se creó, así, en Barcelona una sociedad para comerciar con los países que recorrió la expedición ${ }^{16}$.

También había precedentes lingüísticos a la proposición de ley a un nivel particular. F. García Ayuso fue el filólogo español con una mejor formación en Orientalística del s. XIX ${ }^{17}$. Su interés inicial estaba en la rama indoeuropea de la que estudió sánscrito y avéstico, pero también hebreo, siríaco, etíope, árabe y persa. En 1871, a su regreso de Munich, abrió en Madrid una academia en la que enseñaba estas lenguas

\footnotetext{
12 Gutiérrez Cuadrado, 1985: 82-83. Véase también Álvarez-Pedrosa Núñez, 1994: 61-63.

13 Frase de F. García Ayuso citada en Escribano Martín, 2001: 109.

14 Pérez Díe, 1993: 368.

15 García Figueras, 1947: 12-13.

16 Romero de Tejada, 1980: 45.

17 Álvarez-Pedrosa Núñez, 1994: 55-63.
} 
además de acadio $^{18}$. La circunstancia es importante porque significaba la introducción en España de un establecimiento en que aprender lo que se consideraba entonces el fundamento metodológico de los estudios orientales. Ese mismo año, en su Estudio de la filología en su relación con el sánscrito, hacía la primera descripción correcta en castellano del desciframiento de las escrituras jeroglífica egipcia-que no enseñaba en su escuela ${ }^{19}$ - y cuneiforme mesopotámica. Su personalidad exaltada y su defensa de un catolicismo intransigente con cualquier relectura del texto bíblico a partir de propuestas filológicas o arqueológicas le granjearon la enemistad de los racionalistas. Al mismo tiempo, su actitud arrogante -era joven y se sabía en posesión de unos conocimientos que nadie más tenía en España- le atrajo envidias y recelo por parte del claustro de la Universidad, lo que le privó de la primera cátedra de sánscrito en España en 1877. Desilusionado, fue centrando su actividad en publicar gramáticas de lenguas modernas que servían como libro de texto en su academia y terminó viviendo de la enseñanza del alemán en el Instituto de San Isidro de Madrid.

\section{La proposición de ley de las cátedras de lenguas orientales}

El autor de la proposición de ley fue Miguel Morayta y Sagrario (1834-1917) ${ }^{20}$. Agitador de la vida política española desde su juventud, había iniciado sus actividades como periodista. Durante el Sexenio fue Secretario de la Junta Revolucionaria de Madrid y diputado al Congreso en tres ocasiones. Había obtenido la cátedra de Historia de España en 1868 por oposición, pero no fue resuelta hasta 1874, año en que pasó a ocupar la de Historia Universal. Su programa para la asignatura de Historia Antigua ${ }^{21}$ permite un acercamiento a su concepción de la materia. El contenido de las lecciones dedicadas a Egipto y a Grecia puede conocerse a través de sendos libros dedicados a estas civilizaciones ${ }^{22}$ cuyos capítulos se corresponden con epígrafes del programa. El primero, que transcribe el discurso de apertura del curso 1884-1885 en la Universidad Central, fue atacado por el partido carlista, los obispos y algunos autores católicos ${ }^{23}$, lo que motivó una serie de manifestaciones universitarias en su apoyo conocidas como la Santa Isabel ${ }^{24}$. Las críticas se basaban en dos aspectos: uno histórico, su preferencia por las fuentes egipcias sobre las referencias bíblicas, en especial para cuestiones cronológicas, y otro contemporáneo, su defensa de la libertad de cátedra. La Iglesia consideró ambos ofensivos, en especial el segundo, que veía como un ataque al concordato de 1851, recuperado tras la Restauración ${ }^{25}$. Sus clases

18 Escribano Martín, 2001: 108.

19 La primera obra de lengua egipcia escrita en castellano de la que tengo noticia es la de M. Treviño y Villa, La escritura egipcia y su transcripción castellana en caracteres neo-latinos, publicada en Madrid en 1909.

${ }^{20}$ Pasamar Alzuria, Peiró Martín, 2002: 428-429.

${ }^{21}$ Morayta y Sagrario, 1878.

22 Morayta y Sagrario, 1878-1880, es el libro dedicado a Grecia; Morayta y Sagrario, 1884, el de Egipto.

${ }^{23}$ Por ejemplo, García Ayuso, 1884.

${ }_{24}$ Morayta y Sagrario, 1911.

${ }^{25}$ Molinero Polo, 2011. Sobre el Concordato: Villares, 2008: 26. 
y sus publicaciones sobre el Egipto antiguo nos permiten considerarlo el hombre más cercano a una Egiptología académica española -junto a J.R. Mélida Alinari desde su cargo de Conservador de la Sección Primera, Prehistoria y Edad Antigua, en el Museo Arqueológico Nacional- sin que esto signifique hablar de un egiptólogo en la universidad española, algo de lo que él era consciente si leemos con atención el preámbulo y el texto de la propuesta legal que aquí se analiza ${ }^{26}$. En 1899 fue elegido diputado de nuevo, esta vez como representante por Valencia del partido republicano. Su llegada al Congreso fue turbulenta, según se explicará más adelante. Cuatro meses después presentaba el proyecto de las cátedras de lenguas orientales.

En el Archivo del Congreso se conserva el borrador de la propuesta de ley ${ }^{27} \mathrm{fe}^{\mathrm{f}}$ chado el 7 de noviembre de 1899 y firmado sólo por M. Morayta. Si juzgamos por esta circunstancia y que al final del preámbulo dice que "se honra en presentar..." diríamos que él es el único autor oficial del proyecto. Otra cuestión es si fue inspirado o participó en su concepción alguna persona externa a la institución y que, por esa circunstancia, no podría aparecer en el expediente parlamentario. Pero a falta de documentos adicionales, nada se puede afirmar a este respecto.

Tres semanas después, el 30 de noviembre de 1899, el texto fue leído en el Congreso y, una vez más, sólo interviene M. Morayta en su defensa, según testimonia el diario de sesiones. Él mismo explicó entonces el procedimiento que había de seguirse:

Las propuestas que contienen las dos proposiciones de que se acaba de dar lectura no necesitan apoyo. En todo caso, ambas han de pasar a la Comisión de presupuestos, donde están representadas todas las fracciones y partidos de esta Cámara, y allí se podrá estudiar con detención los fundamentos en que se apoyan y buscar la solución más adecuada. ${ }^{28}$

Mientras que las discusiones sobre el segundo proyecto de ley presentado ese día reaparecen en diversas ocasiones, tanto en el diario de sesiones como en los periódicos del año 1900, el silencio es completo en torno al que nos interesa. Entendemos que tuvo que pasar a la Comisión de presupuestos donde se discutiría, según estaba previsto, en algún momento de los meses siguientes. Sin embargo, según nos comunicaron en el archivo del Congreso, en aquellos años todavía no se hacía un diario de las discusiones en las comisiones, por lo que no sabemos cuáles fueron los argumentos de M. Morayta -si estuvo presente- o los de otros diputados para su defensa o qué se argumentó en contra. Lo único que alcanzamos a conocer, por referencias indirectas, es que en 1900 la ley fue rechazada ${ }^{29}$.

El contenido de la proposición legislativa es el siguiente ${ }^{30}$ :

- Establecimiento en la Facultad de Filosofía y Letras de la Universidad Central de Madrid de tres cátedras, una para lengua egipcia antigua, otra para asiria y una tercera para chino (artículo 1).

\footnotetext{
${ }^{26}$ Sobre su actividad como historiador, véase Wulff Alonso, 2003: 141-147.

27 Es el único documento que incluye el Legajo 313/17.

${ }^{28}$ S.a., 2008: 2362.

${ }^{29}$ Cf. supra, nota 1.

${ }^{30}$ s.a., 2008: Apéndice $7^{\circ}$ al número 66.
} 
- Los beneficiarios habían de ser nombrados en una oposición ante siete catedráticos en el propio centro, dejando a éste decidir la convocatoria, las condiciones y la forma de la oposición (artículo 2).

- Los requisitos previos eran haber obtenido una licenciatura en Filosofía y Letras y tener entre 21 y 26 años (artículo 3 ).

- Los opositores ganadores tendrían que adquirir la formación lingüística en sus respectivas lenguas durante una estancia de tres años en el extranjero: al menos uno completo en Francia y el resto en Inglaterra y Alemania. Al decano de la Facultad de Filosofía y Letras correspondía el control de sus estudios a partir de las memorias que entregarían semestralmente (artículos 4 y 6).

- Se fijaba la cuantía de la pensión como becarios y se especificaba que sólo al regreso de esa estancia y tras la aprobación del oportuno informe sobre su aprendizaje, los beneficiarios entrarían a formar parte del cuerpo de catedráticos. A partir de ese momento explicarían la asignatura en dos cursos y días alternos (artículo 5).

Pueden caber dudas acerca de si la obligatoriedad de residir en Alemania, Francia e Inglaterra, idéntica para los tres becarios, con independencia de la lengua que aprendan, se debía a un conocimiento de la valía real de las escuelas lingüísticas de estos países y de los profesores que las integraban o era más bien para crear un marco que permitiera amplitud de contactos profesionales y el conocimiento de las lenguas modernas que pudieran serles útiles para el futuro.

Resulta también interesante reconocer por qué M. Morayta planteaba que las tres cátedras se creasen en un único centro universitario, a pesar de defender desde el inicio del preámbulo la importancia de que el país contase con especialistas en estos campos:

Debiendo ser libre su estudio, habrán de ser pocos los que las estudien: basta y sobra con que cada año salgan de la Universidad dos o tres que las conozcan y propaguen.

Hay que recordar que en el Real Decreto de 1900 -un año después de la proposición que nos ocupa-, sólo se establecieron cuatro universidades con sección de Historia $^{31}$, y el doctorado se cursaba únicamente en la de Madrid.

Como ya se ha señalado, no hay documentación que permita saber cómo se argumentó el rechazo de la propuesta de ley. Sin embargo, se pueden sugerir algunas hipótesis.

La primera consideración es la más obvia. La propuesta se presentó en noviembre de 1899, sólo un año después de la pérdida de las últimas colonias, en el momento más grave de la crisis económica y bajo un cierto desánimo generalizado. Algunos dirían que no eran éstas las circunstancias más apropiadas; otros argumentarían que los periodos de crisis son los más indicados para tomar nuevos rumbos. M. Morayta es consciente de la situación nacional, pero también se adelanta a las críticas exponiendo una defensa:

Conviene, además, en estos tiempos de penuria, limitarse a lo más preciso, si bien no hay gasto superfluo posible, si se emplea bien, en la enseñanza pública.

31 En Madrid, Sevilla, Zaragoza y Valencia (Pasamar Alzuria, Peiró Martín, 1987: 35). 
Una segunda hipótesis es que el rechazo fuera consecuencia de una posible animadversión hacia M. Morayta en el Congreso. Su llegada a la Cámara fue motivo de una dura discusión durante varias sesiones pues un periódico, El Nacional, recordó bajo el titular "Fuera traidores" su patrocinio de los comités hispano-filipinos que pudieron servir de apoyo a intereses de los independentistas del archipiélago asiáti$\mathrm{Co}^{32}$. A causa de la disputa, tardó una semana en ser proclamado diputado. Un mes después, cuando aún no se habían calmado los ánimos, presentaba una proposición de ley contra las instituciones religiosas fundadas después de 1837, lo que tuvo que profundizar la animadversión que sentían por él los neocatólicos -recuérdese la controversia tras su discurso de 1884-; acerca de este proyecto, Fernando Soldevilla, periodista de ideas liberales moderadas, señalaba en su anuario político:

Esta proposición habría causado algún efecto si hubiera sido presentada por persona de más autoridad y prestigio que el Sr. Morayta pero a este señor se le hacía poco caso en la Cámara. ${ }^{33}$

Sin embargo, a pesar de estas palabras, el diario de sesiones de ese año y el siguiente muestra que M. Morayta fue un político muy activo, que presentó un alto número de proposiciones de ley, algunas en solitario y otras con más diputados, en ocasiones en cabeza de la lista, lo que indica una cierta capacidad de liderazgo dentro de su grupo o, más ampliamente, de su ideología política. En estos mismos años, los periódicos reseñan su asistencia a banquetes de homenaje tanto a aristócratas como a artistas, con frecuencia ocupando un lugar de honor, lo que muestra también un cierto prestigio mundano. A pesar de esto, cabe preguntarse si sus anteriores disputas con la Iglesia y con los partidos más conservadores pudieron provocar cierto desinterés sobre la proposición misma.

El último de los argumentos es más bien una interrogación pero puede ser el más duro de responder desde la posición de la Orientalística actual, pues consiste en plantear si el rechazo al proyecto de ley puede ser considerado un baremo del desinterés de la sociedad española finisecular hacia el Orientalismo antiguo o sólo de los representantes de los dos partidos mayoritarios entonces en la Cámara. Hay que reconocer que es poco probable que pueda surgir un interés hacia aquello que apenas se conoce y la sociedad hispana no tenía las oportunidades que había en otros países de nuestro entorno. La producción bibliográfica propia sobre Oriente era extremadamente escasa $^{34}$ como también las traducciones de otras lenguas. Las colecciones privadas de antigüedades orientales eran pequeñas, las públicas poco numerosas; en museos de Madrid, Barcelona, Vic, Vilanova i la Geltrú o San Sebastián tenemos constancia de la entrada de donaciones desde fondos particulares antes del cambio de siglo, pero apenas sabemos si fueron expuestas de manera visible para el público, salvo en el

32 Soldevilla, 1900: 180.

33 Soldevilla, 1900: 234.

${ }^{34}$ Análisis de algunos autores y obras específicas sobre el Egipto antiguo en: Molinero Polo, 2004: 35-40 y Molinero Polo, 2011: 138-141 y 144-145. Para los investigadores españoles del s. XIX que trabajaron en Oriente Próximo, véanse los artículos reunidos en Córdoba Zoilo, 2005: 745-858. 
Museo Arqueológico Nacional ${ }^{35}$. La moda egiptizante en arquitectura, decoración, joyas y hasta música estaba presente ${ }^{36}$, pero hay que preguntarse hasta qué punto era un reflejo del interés por el Egipto antiguo o, más bien, por la propia egiptomanía desarrollada en otros países. Y, sobre todo, el Orientalismo español, como ya se ha puesto de relieve en numerosos estudios, se dirigió preferentemente al Magreb ${ }^{37}$; así, mientras algunos arquitectos becados en Roma se desplazaban a Grecia o a Egipto, los pintores viajaban al noroeste africano ${ }^{38}$.

\section{Las consecuencias del rechazo de la proposición de ley}

En 1900, el recién creado Ministerio de Instrucción Pública y Bellas Artes reorganizaba por Real Decreto la Facultad de Filosofía y Letras ${ }^{39}$. Introducía la especialización al crear tres secciones: Filosofía, Historia y Letras; fundía los estudios de la Escuela Superior de Diplomática con los de Historia; racionalizaba algunos aspectos del profesorado. Además, aunque en la especialidad de Letras solamente se concedía atención al castellano, por primera vez el decreto ministerial se refiere a otras lenguas.

Enriquecida la Facultad con estos estudios, era urgente, más que nunca, el establecimiento de otros no menos imprescindibles. Seguramente que, de contar con los recursos necesarios, se habría concedido en esta reforma el lugar que corresponde a la Egiptología y Asiriología, así como también a las lenguas y literaturas célticas, que tan de cerca nos tocan, y a la lengua y literaturas germánicas, cuyo conocimiento es cada día más útil y provechoso.

Propónese, sin embargo, el exponente, atender, más adelante, a tan justas exigencias científicas, enviando al extranjero, si necesario fuere, pensionados que cultiven estos estudios tan florecientes en otras naciones, para poder enseñarlos luego en el periodo más adecuado de la carrera de Letras.

La mención a las disciplinas orientales en el marco lingüístico y no en el histórico es una herencia directa de la iniciativa de M. Morayta, así como el propósito de becar en el extranjero a los futuros profesores de estas asignaturas. La propia referencia a la necesidad de esperar a una mejora de la situación económica para crear el marco institucional para la Egiptología y la Asiriología es una confirmación indirecta de que la principal razón para que no se aceptase, unos meses antes, la proposición en la Comisión del Congreso fue la pecuniaria; la ideología política del proponente parece, así, quedar al margen del rechazo, pues el decreto que recoge estos propósitos estaba promovido por Antonio García Alix, ministro en el gobierno conservador de Antonio Silvela.

35 Un listado, no exhaustivo, de donantes al Museo Arqueológico Nacional, como ejemplo de colecciones españolas, en Molinero Polo, 2004: 60-61; sobre sus coleccionistas, Pérez Díe, 1993 y Molinero Polo, 2004: 20-23. Sobre la exhibición de aegyptiaca en la residencia madrileña del marqués de Cerralbo: Molinero Polo, Jaramago y García Fernández, en prensa.

${ }^{36}$ Saguar Quer, 1997; Sevilla Cueva, 2001.

${ }^{37}$ Morales Lezcano, 1990.

38 VV.AA., 1988.

39 Gaceta de Madrid, 22 de julio de 1900: 310-311. 
La afirmación que inició la búsqueda a partir de la cual se ha escrito este artículo -la proposición de ley como causa de la pérdida del interés de J.R. Mélida por el Egipto antiguo- puede responderse ahora parcialmente ${ }^{40}$. En 1899, superaba los 40 años por lo que no habría podido presentarse a las oposiciones de estas cátedras, de haberse aprobado la ley, pues el límite estaba fijado en los 26. A falta de documentos, nada puede concluirse sobre una posible relación entre el conservador del Museo Arqueológico Nacional y M. Morayta que hubiera influido en la cuestión de la edad o en otras. De ambos se ha señalado que en uno u otro momento estuvieron cercanos a la Institución Libre de Enseñanza ${ }^{41}$. No se ha publicado ningún documento que pueda hacer pensar en una animadversión entre ellos o en un intento malintencionado de alejar al arqueólogo de la cátedra de lengua egipcia. El único indicio podría ser la afirmación de J. de D. de la Rada de que "en España (...) no hay tales enseñanzas [de Egiptología y] Mélida ha emprendido por sí sólo, el difícil estudio de aquellas"42. Pronunciada en 1899 , cabe la posibilidad de que la frase ponga de manifiesto un cierto distanciamiento entre los profesores de la Escuela Superior de Diplomática - de la que era catedrático aquél- y la Universidad, pues si bien es cierto que en ésta no había enseñanzas regladas específicas de Egiptología, el país del Nilo estaba incluido en las clases de M. Morayta en su asignatura de Historia Antigua y éste le dedicó varias publicaciones durante las dos décadas anteriores que en la frase anterior se olvidan ${ }^{43}$; pero también es posible que se trate sólo de una exageración amable motivada por el propio carácter laudatorio del discurso en que se pronunciaron aquellas palabras. No obstante, las aseveraciones de algunos investigadores sobre la desilusión de J.R. Mélida no pueden olvidarse, a pesar de que no han aportado las fuentes explícitas en que se apoyan; el carácter lingüístico de la cátedra podría ser una de las causas adicionales de esa frustración si ésta pudiera confirmarse. Por otra parte, ese abandono de la Egiptología por J.R. Mélida no fue completo, pues en las décadas posteriores a 1900 siguió presentando trabajos egiptológicos $^{44}$ y parece que se interesó por enviar a algún estudiante a aprender arqueología

${ }^{40}$ Cf. supra, n. 2.

${ }^{41}$ Para M. Morayta: Wulff Alonso, 2003: 141. Respecto a J.R. Mélida: Almela Boix, 2004: 261-262.

${ }^{42}$ Citado por Almela Boix, 2004: 263. En el mismo año de la proposición de ley, el Diccionario Enciclopédico Hispano Americano... describía a J.R. Mélida de este modo: "su especialidad es el conocimiento de las antigüedades egipcias y griegas. Es el único egiptólogo que tenemos en España, y los trabajos de interpretación de los jeroglíficos que, según se dice, practica en el Museo, le asegurarán ese título" (VV.AA., 1899: 386). Las voces de este diccionario no van firmadas, por lo que no podemos saber quién escribió ésta; no obstante, resulta llamativo que en el mismo año en que se propuso la ley de cátedras de lenguas - que habría excluido al arqueólogo por su edad y por su especialidad-, esta frase dejaba entender que él tenía esos conocimientos lingüísticos, que no podemos confirmar por otros medios. Casado Rigalt (2006: 95) afirma que no leía escritura jeroglífica.

43 Véase n. 19. Sobre sus trabajos de Historia antigua de Egipto: Wulff Alonso, 2000; Molinero Polo, 2011: 138-141.

${ }^{44} \mathrm{Su}$ producción en este campo fue más divulgadora que de especialista. Antes del cambio de siglo, había publicado dos artículos en sendas revistas académicas (en 1881 y 1898), dos libros, uno muy breve sobre religión (en 1884) y otro de arte (1897), unos artículos en periódico sobre la colección de El Louvre (1884) y había leído dos conferencias sobre arte y pirámides (1886 y curso 1889-1890). Tras la proposición de ley publicaba un artículo (1907), un capítulo largo sobre arte (1933), el prólogo a la obra de J. Cascales (de 1910), dos reseñas a libros egiptológicos (1910 y 1928) y pronunciaba cuatro conferencias en 1909. Para estas obras y actividades, véase Casado Rigalt, 2007, unas en el listado bibliográfico final, otras en el propio texto. Resulta sorprendente que no se encargue a J.R. Mélida la 
egipcia en El Cairo, como se verá más adelante. En la reducción cierta del ritmo de su producción de temas egipcios pudieron intervenir otros factores como su interés por campos de investigación más cercanos y que absorberían su tiempo: la cultura ibérica (de la que había empezado a publicar en la década de 1890) ${ }^{45}$, las excavaciones de Emerita Augusta y las de Numancia. No puede obviarse que también en los años del cambio de siglo abandonó su faceta literaria, a la que había dedicado una decena de novelas en las dos décadas anteriores ${ }^{46}$.

Así, a partir de la documentación accesible, la impresión es que en el tema del límite de edad, se intentó actuar con coherencia en el proyecto legislativo: una persona joven tenía una carrera por delante y una mayor posibilidad de establecer una escuela y por eso se planteó tan bajo. No podemos olvidar las consecuencias desafortunadas de las varias oposiciones recientes a la cátedra de sánscrito y es posible que esta amarga enseñanza estuviera en la mente del legislador en el momento de la redacción.

A través de la proposición de ley, M. Morayta se muestra consciente del camino hacia la especialización que había emprendido la Historia. Él, que era un historiador generalista y escribía unas veces sobre el Egipto antiguo y otras sobre la Revolución Francesa, que podía transitar en sus estudios entre China y la Península Ibérica, solicitó la creación de cátedras para una docencia muy específica. Esta situación era la consecuencia de la paulatina asimilación en España de los principios de la escuela positivista. La primacía que tenían para ésta los documentos escritos como fuente inmediata y "objetiva" para la reconstrucción histórica se manifiesta en que la proposición pretende crear cátedras de Filología, no de Historia, dando así prioridad a la lectura de los textos en su lengua original. Podría hablarse de un cierto olvido -o aún desconocimiento- de las posibilidades de la Arqueología como herramienta para reconstruir el pasado, la especialidad que el propio J.R. Mélida estaba colaborando a desarrollar en España, al depositar en los filólogos la responsabilidad de crear la Orientalística española.

La proposición de ley se enmarca también en un aspecto significativo del desarrollo de la profesionalización de los historiadores, que es el viaje de estudios al extranjero. En 1843, había partido el primer becario español del s. XIX que traspasaba las fronteras para ampliar estudios, un profesor interino de Filosofía ${ }^{47}$. En torno a 1880, varios profesionales de la Historia habían profundizado sus conocimientos sobre métodos de investigación en centros europeos ${ }^{48}$. No se trataba, por tanto, de una novedad en el panorama universitario. Al mismo tiempo, era una iniciativa que se inscribía en un modelo de adquisición de conocimientos que estaba presente en la Egiptología internacional. Tras varios años de estancia en la Universidad de Berlín, bajo la dirección de Adolf Erman (catedrático entre 1884 y 1923) y de su sucesor Kurt Sethe (de 1923 a 1934), sus alumnos Walter E. Crum (en Alemania en torno

redacción de las voces sobre cultura egipcia en el Diccionario Enciclopédico Hispano Americano... , que escribió Francisco Fernández y González, aunque sí se encargaría de las de cultura material, pues él asume la "Arqueología oriental y clásica".

${ }^{45}$ Casado Rigalt, 2006: 137-176.

${ }^{46}$ Casado Rigalt, 2006: 63-73.

47 Se trata de Julián Sanz del Río, cuyo nombramiento para la Facultad de Filosofía de la Universidad Central implicaba la obligación de ampliar en Alemania sus conocimientos de historia de su disciplina y estudiar las causas del florecimiento científico en este país (Jiménez, 1971: 319-322).

48 Pasamar Alzuria, Peiró Martín, 1987: 20. 
a 1890), Boris A. Turaieff (en la década de 1890), George A. Reisner (de 1893 a 1896), James H. Breasted (1894), Gustave Jéquier (década de 1890), Alan Gardiner (de 1902 a 1912) y Wolja Erichsen (1925 a 1944), regresaron a sus países de origen (Gran Bretaña, Rusia, Estados Unidos, Suiza, Dinamarca) y dieron nacimiento en ellos a sus primeras escuelas de lingüística egipcia y, en sentido más amplio, de Egiptología ${ }^{49}$. Podemos, así, convenir que la proposición de ley española ponía unas bases correctas que estaban funcionando coetáneamente; que de éstas se hubieran podido obtener idénticos resultados en España depende de otros factores que no se pueden incorporar a este análisis. Es importante recordar que los dos catedráticos berlineses eran expertos lingüistas y que el método de la crítica textual era la base para sus estudios históricos y la que legaron a sus alumnos -lo que ha marcado considerablemente el desarrollo de la disciplina durante la mayor parte del s. XX-, coincidiendo con el planteamiento que suponemos a M. Morayta para su decisión de destinar las becas a estudios de lenguas.

Una derivación de las propuestas legislativas que se revisan en este artículo es un tercer intento documentado para activar el aprendizaje de la Egiptología en el seno de una institución académica. Tras el Segundo Congreso Internacional de Arqueología, celebrado en El Cairo en la primavera de 1909, J.R. Mélida presentó un informe a la Real Academia de la Historia como delegado de esta institución y del propio gobierno español en el evento ${ }^{50}$. Una semana más tarde, el 11 de junio, el Secretario de la Academia escribía una carta al Ministro de Instrucción Pública y Bellas Artes, Faustino Rodríguez Sampedro ${ }^{51}$, solicitándole, según Casado Rigalt, "que España tuviese un representante que asistiera a alguno de los institutos arqueológicos con el fin de que se adoctrinase [en] las enseñanzas egiptológicas" - se refiere a los institutos europeos ya abiertos en Egipto en ese momento, es decir, el francés o el alemán-. Este investigador atribuye a J.R. Mélida la autoría intelectual de la propuesta, que habría estado apoyada por Antonio Sánchez-Moguel. Sin embargo, no hay noticia de que ningún becario español viajara a Egipto con ese cometido en los años siguientes, por lo que es evidente que la solicitud no tuvo el efecto requerido. Es significativo reconocer la modificación metodológica que supone esta propuesta, pues el beneficiario se especializaría en Arqueología, no en lengua como en las propuestas anteriores, de acuerdo a los intereses del inductor de la solicitud. Adicionalmente, esto vendría a señalar que no había, por parte de J.R. Mélida, un olvido tan completo respecto a Egipto, tal vez porque el interés que le había suscitado la cultura ibérica desde fines del s. XIX y la influencia de Oriente en las creaciones plásticas que se estaban descubriendo en sus yacimientos hacía más urgente, si cabe, el nacimiento de especialistas españoles en la producción material del Mediterráneo oriental.

Una consecuencia indudable del rechazo al proyecto de las cátedras -y a la solicitud del secretario de la Real Academia de la Historia- fue el retraso en el desarrollo

${ }^{49}$ Véanse las entradas biográficas de todos los egiptólogos mencionados en el párrafo en: Dawson, Uphill, Bierbrier, 1995.

${ }^{50}$ Fita, 1909: 368.

${ }^{51}$ Casado Rigalt, 2006: 134-135. No menciona dónde se conserva esta carta o si su fuente es algún otro tipo de documento, lo que ha hecho imposible contrastar o ampliar la información que él mismo proporciona. 
de una Orientalística española con respecto a los países de nuestro entorno. Además de recordar una simple evidencia, con esta aseveración se pretende abrir un debate sobre la importancia que las intervenciones privadas en suelo oriental, más allá del aura de pioneras y aventureras, han tenido en el surgimiento de la disciplina en España. Si tomamos como modelo el caso egipcio, que es el que tiene unos precedentes más claros, podemos constatar que en las décadas en torno al cambio de siglo se produjeron varias actuaciones puntuales realizadas por particulares, "precursores" si utilizamos el calificativo, que entendemos correcto, con que J. Padró ha calificado al segundo personaje de esta relación ${ }^{52}$ :

- Antonio Amador de los Ríos trabajó algunos meses como colaborador del Marqués de Rochemonteix en su primera campaña epigráfica en el templo de Edfú; en su caso, en el marco de una beca oficial concedida para el estudio de las obras arquitectónicas.

- Eduard Toda i Güell, cónsul español en Egipto, vigiló la extracción del ajuar de la tumba de Sennedjem en Deir el Medina y, lo que es más importante, publicó la decoración y sus textos. Formó, además, una de las mayores colecciones egiptológicas que han llegado a España. A él se refiere, sin duda M. Morayta en el preámbulo de la proposición de ley, cuando menciona triste es decirlo, si en alguna Memoria o libro español se leen afirmaciones que parecen indicar algún conocimiento del asirio o del egipcio, tal erudición, si no de segunda mano, es debida casi siempre a la buena amistad de algún extranjero ${ }^{53}$.

- Vicente de Galarza y Pérez Castañeda, profesor de filosofía en la Universidad Egipcia de El Cairo, detentó la concesión arqueológica de una colina en Guiza cuya excavación fue dirigida por Ahmed Kamal y Georges Daressy. Entre 1907 y 1909 descubrieron varias tumbas del Reino Antiguo, entre las que se encuentra la de una hija y esposa real de la dinastía IV ${ }^{54}$.

Estas intervenciones suelen ser presentadas como el fundamento de la Egiptología española. Pero, sin menospreciar el valor de estas tareas, sería conveniente reubicarlas historiográficamente. Sus autores trabajaron en Egipto durante un periodo muy breve (los dos primeros en única campaña y el conde de Galarza en una de dos años), desde una posición externa (A. Amador colaboró con un epigrafista; E. Toda acompañaba como amigo a los arqueólogos; $\mathrm{V}$. de Galarza financió la excavación que le fue concedida) y sólo el segundo escribió varias obras sobre la civilización faraónica durante un lustro. Sin continuidad ni discípulos. Y sin estas dos consecuencias, cada trabajo se convierte en un esfuerzo individual y aislado.

Si hubiera que buscar unos fundamentos a la Egiptología española actual, habría que plantear otras bases, ligadas a la profesionalización de la disciplina y su transmisión en el seno de instituciones oficiales ${ }^{55}$. Naturalmente, ésta podría no haber sido la

${ }^{52}$ Padró, en Toda, s.d.: XII.

53 Toda, s.d. [1887]: 63: Urbain Bouriant se encargó de "colacionar los textos e interpretar su sentido" para la publicación del cónsul español.

54 Trad, Halwagy, 2009. Dos de las estatuas encontradas en la excavación pueden verse en el catálogo en que se incluye el anterior artículo, pp. 176-179.

${ }^{55}$ La afirmación de J. de D. de la Rada acerca de sus propios esfuerzos para establecer las enseñanzas de Egiptología y Asiriología en España (citada por Almela Boix, 2004: 263) no ha sido investigada con más detalle. Al haber sido maestro de J.R. Mélida, el vínculo entre ambos podría plantearse como 
única vía; desde Inglaterra, una fundación privada, la Egypt Exploration Society ${ }^{56}$, ha tenido una importancia excepcional en el desarrollo de una arqueología científica en Egipto, así como en el establecimiento de la Egiptología en su país, pero en España no surgió ningún organismo comparable ${ }^{57}$.

Una primera base estaría formada por investigadores sobre Oriente ligados a la Iglesia católica, si es que la circunstancia de la pertenencia a ésta de varios autores o la cercanía de creencias a ella permiten hablar de una línea de continuidad, lo que también habría de probarse. En el caso egipcio, partiendo de Manuel de la Peña y Fernández (su Manual de Arqueología Prehistórica es de 1890) y de Felix Rougier (Biblia y Egiptología, 1893), pasando por las colecciones reunidas por sacerdotes como las que forman la base del Museo del Oriente Bíblico de Montserrat y de otros en su entorno (Tarragona, Palma de Mallorca, nacidos en la primera parte del s. XX), llegamos a los trabajos del Padre Benito Celada que se prolongan hasta la década de $1960^{58}$. Si bien el número de autores es limitado, como también lo es su producción bibliográfica, parecen mantener una línea epistemológica comparable y transmitida de unos a otros.

La segunda sería la que surge a partir de la Misión Arqueológica Española en Nubia. De la labor docente de sus participantes o del estudio de las piezas donadas por Egipto y Sudán en las instituciones que las custodian desciende la mayor parte de la actual generación de egiptólogos españoles. En las excavaciones y actividades epigráficas participaron, entre otros, Manuel Presedo o Jesús López, que han tenido sus discípulos en los centros en que enseñaron, el primero como catedrático de Historia Antigua, el segundo como profesor visitante. Aquellas campañas tuvieron su continuidad en la Misión Arqueológica Española en Egipto, que trabaja en Ihnasya al-Medina, otro de los fundamentos de la disciplina en nuestro país. Y en agradecimiento a la labor realizada y a los fondos donados a Egipto para el salvamento de Abu Simbel, se recibió el templo de Debod, además de las antigüedades que componen la colección nubia del Museo Arqueológico Nacional, donde trabajan o se han formado otra parte de los egiptólogos activos. En todos estos casos, hablamos de instituciones oficiales como base de una Egiptología que es, por definición, académica. Así, irónicamente, el propósito de M. Morayta se va alcanzando por una vía semejante a la

un nacimiento de "escuela" egiptológica; sin embargo, éste no tuvo discípulos en esta disciplina y la transmisión quedaría, así, rota. También hay que tener en cuenta que relacionados con el Egipto antiguo, J. de D. de la Rada sólo escribió el capítulo de Alejandría en el relato del viaje de la fragata Arapiles y tres artículos muy breves, lo que no parece una producción bibliográfica que avale aquella afirmación. Se excluye de este inventario sus trabajos relacionados con las falsificaciones egiptizantes del Cerro de los Santos.

56 Desde 1882, Society for the Promotion of Excavation in the Delta, nacida con el propósito de obtener información sobre la presencia hebrea en Egipto siguiendo la información de la Biblia; pronto fue convertida en Egypt Exploration Fund; el nombre actual data de 1919 (Reid, 2002: 178). Véase también James, 1982, cuyo capítulo sobre los inicios de la Sociedad, por M. Drower, resulta un tanto autocomplaciente y tópicamente antifrancés.

${ }^{57}$ Una visión más favorable a la actuación de una asociación egiptológica en: López Grande, 1997. Hay que tener en cuenta que este artículo tiene ya quince años y en ese tiempo la situación profesional y la actividad didáctica e investigadora de algunos egiptólogos en las universidades españolas y en el CSIC se ha afianzado, lo que ha cambiado significativamente la ponderación respecto a aquel momento.

58 Para una relación más detallada, véase Molinero Polo, 2011: 143-145. 
que él había propuesto, aunque las circunstancias para su concreción hayan sido muy diferentes.

* Este trabajo se ha redactado en el marco del proyecto de investigación HAR 2011-25292, La "Historia de las religiones" y el "estudio de las religiones" en España antes del Concilio Vaticano II, financiado por el Ministerio de Ciencia e Innovación y los FEDER.

\section{Bibliografía}

Almela Borx, A. (1991): “La aportación de José Ramón Mélida a la consolidación de la Arqueología como disciplina científica en España”, en R. Olmos y J. Arce (coords.): Historiografía de la Arqueología y de la Historia antigua en España (siglos XVIII-XX), Instituto de Conservación y Restauración de Bienes Culturales, Madrid: 131-134.

Almela Borx, A. (2004): “José Ramón Mélida Alinari”, en M. Ayarzaguena Sanz y G. Mora Rodríguez (coords.): Pioneros de la arqueología en España. Del siglo XVI a 1912, (Zona Arqueológica 3), Museo Arqueológico Regional, Alcalá de Henares: 261-268.

Álvarez-Pedrosa NúÑEz, J.A. (1994): "La lingüística indoeuropea en España hasta 1930", Revista Española de Lingüistica 24/1: 49-67.

Casado Rigalt, D. (2006): José Ramón Mélida (1856-1933) y la Arqueología española, Real Academia de la Historia, Madrid.

Casado Rigalt, D. (2007): “José Ramón Mélida y la Egiptología en España (18751925)", Boletín de la Asociación Española de Egiptología 17: 23-38.

Chinchilla Gómez, M. (1993): "Viaje a Oriente de la fragata Arapiles", en A. Marcos Pous (coord.): De Gabinete a Museo. Tres siglos de Historia, Ministerio de Cultura, Madrid: 286-299.

Córdoba Zoilo, J.Ma. (ed.) (2005): Españoles en Oriente Próximo (1166-1926). Aventureros y peregrinos, militares, cientificos y diplomáticos olvidados en el redescubrimiento de un mundo. (Arbor CLXXX / 711-712), Madrid.

David, E. (1994): Mariette Pacha. 1821-1881, Pygmalion, Paris.

Dawson W.; Uphill, E.P.; Bierbrier, M.L. (1995, 3rd ed.): Who was who in Egyptology, The Egypt Exploration Society, London.

Escribano Martín, F. (2001): "Los estudios sobre Oriente en la España de finales del siglo XIX: la vida y obra de Francisco García Ayuso", en J.Ma Córdoba Zoilo; R. Jiménez Zamudio, C. Sevilla Cueva (eds.): Actas del primer seminario monográfico de primavera. El redescubrimiento de Oriente Próximo y Egipto. Viajes, hallazgos e investigaciones, Universidad Autónoma de Madrid, Madrid, 107-116.

EsCRIBANo Martín, F. (2005): "Adolfo Rivadeneyra, un diplomático español al servicio del estudio y del viaje por Oriente", Arbor CLXXX / 711-712: 789-804.

FitA, F. (1909): "Noticias", Boletín de la Real Academia de la Historia LV/IV: 368. 
García Ayuso, F. (1884): Cabos sueltos de historia o hechos importantes de la historia y de las tradiciones de los pueblos, examinados a la luz de los descubrimientos modernos, Sucesores de Rivadeneyra, Madrid.

García Figueras, V. (1947): Don Juan Víctor Abargues de Sostén, explorador de Abisinia. (Una figura poco conocida de nuestros exploradores africanos del Siglo $X I X)$, Conferencia pronunciada en el Instituto de Estudios Africanos el día 30 de Abril, Texto mecanografiado conservado en la Biblioteca Nacional de España.

García SÁnchez, J. (2004): “Arquitectos españoles del siglo XIX en Grecia y Egipto", Academia. Boletín de la Real Academia de Bellas Artes de San Fernando 98/99: 53-72.

García Tudela, I. (1873): "Extracto del diario de navegación del comandante de la fragata Arapiles, correspondiente al viaje verificado por dicho buque al interior del Mediterráneo desde el día 7 de julio hasta el 22 de setiembre de 1871", Anuario del depósito hidrográfico XI: 1-56.

Gutiérrez Cuadrado, J. (1985): "La lengua a debate", en J.L. Peset et alii: Pasado, presente y futuro de la universidad española. (Serie Universitaria 225), Fundación Juan March, Madrid: 68-90.

James, T.G.H. (1982): Excavating in Egypt. The Egypt Exploration Society 18821982, British Museum, London.

JiMÉNEZ, A. (1971): Historia de la Universidad Española, Alianza, Madrid.

LóPez Grande, Ma.J. (1997): "Los estudios de Egiptología en España y la Asociación Española de Egiptología", en G. Mora, M. Díaz-Andreu (eds.): La cristalización del pasado. Génesis y desarrollo del marco institucional de la Arqueología en España, Servicio de Publicaciones de la Universidad de Málaga, Málaga: 721-728.

LóPEZ-Ocón, L. (2003): "La Comisión Científica del Pacífico: de la ciencia imperial a la ciencia federativa", Bulletin de l'Institut Français des Études Andines 32/3: 479-515.

Marcos Pous, A. (1993): "Origen y desarrollo del Museo Arqueológico Nacional", en A. Marcos Pous (coord.): De Gabinete a Museo. Tres siglos de Historia. Ministerio de Cultura, Madrid: 21-99.

Molinero Polo, M.Á. (2004): "El pozo y el péndulo. La actividad egiptológica de anticuarios y arqueólogos españoles, 1868-1966", en A. Martín Flores y Ma V. López Hervás (coords.): Españoles en el Nilo. I. Misiones Arqueológicas en Egipto, Museo de San Isidro, Madrid: 15-62.

Molinero Polo, M.Á. (2011): "El Egipto antiguo en la controversia académica española del siglo XIX. El discurso de Miguel Morayta en la Universidad Central, octubre de 1884", Bandue. Revista de la Asociación Española de Ciencias de las Religiones 5: 131-150.

Molinero Polo, M.Á.; Jaramago, M. y García Fernández, G. (en prensa): "El marqués de Cerralbo y el coleccionismo de antigüedades egipcias en España durante la segunda mitad del s. XIX", en J. das C. Sales, L. M. de Araujo y R. Sousa (eds.): IV Congresso Ibérico de Egiptologia / IV Congreso Ibérico de Egiptología, Lisboa. 
Morales Lezcano, V. (1990): Africanismo y Orientalismo español en el s. XIX, Instituto de Cooperación con el Mundo Árabe / UNED, Madrid.

Morayta y Sagrario, M. (1878): Programa de Historia Universal conforme a las explicaciones del Catedrático de esta asignatura en la Facultad de Filosofia y Letras de la Universidad de Madrid Doctor Don... Madrid.

Morayta y Sagrario, M. (1878-1880): Historia de la Grecia antigua, F. Góngora, Madrid.

Morayta y Sagrario, M. (1884): Discurso leído en la Universidad Central en la solemne inauguración del curso académico 1884-1885 por el doctor Don... Tipografía de Diego Pacheco, Madrid.

Morayta y Sagrario, M. (1911): La libertad de la Cátedra. Sucesos universitarios de la Santa Isabel, Editorial Española-Americana, Madrid.

Pasamar Alzuria, G.; Peiró Martín, I. (1987): Historiografia y práctica social en España, Universidad de Zaragoza, Zaragoza.

Pasamar Alzuria, G.; Peiró Martín, I. (2002): Diccionario Akal de historiadores españoles contemporáneos (1840-1980), Akal, Madrid.

Pérez Díe, Ma . C. (1993): "Las colecciones egipcias y el Próximo Oriente", en A. Marcos Pous (coord.): De Gabinete a Museo. Tres siglos de Historia, Ministerio de Cultura, Madrid: 159-169.

ReID, D.M. (2002): Whose Pharaohs? Archaeology, Museums, and Egyptian National Identity from Napoleon to World War I, University of California Press, Berkeley, Los Angeles, London.

Romero de Tejada, P. (1980): "Los españoles del siglo XIX en África y las colecciones del Museo Nacional de Etnología", en Actas del I Congreso Español de Antropología, Barcelona: vol. II, 39-62.

S.A. (2008): Diario de sesiones del Congreso de los Diputados. Legislaturas de 1899 a 1900, Congreso de los Diputados, Madrid.

SAGUAR Quer, C. (1997): "Egiptomanía y arquitectura en España (1840-1940)”, Goya 259-260: 386-406.

Sevilla Cueva, Ma . C. (2001): "Egipto y España en la música: el caso de Aida y La Corte del Faraón", en J.M ${ }^{a}$ Córdoba Zoilo; R. Jiménez Zamudio, C. Sevilla Cueva (eds.): Actas del primer seminario monográfico de primavera. El redescubrimiento de Oriente Próximo y Egipto. Viajes, hallazgos e investigaciones, Madrid, 355378.

Soldevilla, F. (1900): El año político 1899, Imprenta Enrique Fernández de Rojas, Madrid.

TodA, E. (s.d.): Son Notém en Tebas. Sennedyem en Tebas. (Orientalia Barcinonensia 10). Prólogo, apéndice y fotografías por Josep Padró. Sabadell, Ausa [Edición facsímil de: Son Notem en Tebas. Inventario y textos de un sepulcro egipcio de la XX Dinastía, Establecimiento tipográfico de Fortanet, Madrid, 1887]. 
Trad, A.; el Halwagy, M. (2009): "La tumba 'Galarza”", en Ma C. Pérez Díe (ed.): 120 años de arqueología española en Egipto, Sociedad Estatal de Conmemoraciones Culturales, Madrid: 35.

Villares, R. (2008): “Alfonso XII y Regencia”, en R. Villares y J. Moreno Luzón, Historia de España. Volumen 7. Restauración y Dictadura, Crítica / Marcial Pons, Barcelona / Madrid: 1-304.

VV.AA. (1899): Diccionario Enciclopédico Hispano Americano de literatura, ciencias y artes. Tomo XXV. Apéndice H-Z, Montaner y Simón, Barcelona.

VV.AA. (1988): Pintura Orientalista Española (1830/1930), Fundación Banco Exterior, Madrid.

Wulff Alonso, F. (2000): "En los orígenes de la Historia social de la Antigüedad en España: 'Las clases trabajadoras en la Antigüedad' de M. Morayta (1886)", Baetica. Estudios de Arte, Geografía e Historia 22: 333-357.

WulfF Alonso, F. (2003): Las esencias patrias. Historiografia e Historia antigua en la construcción de la identidad española (siglos XVI-XX), Crítica, Barcelona. 